\title{
Clinical implications of transpterygoid approach to mandibular nerve
}

\author{
Ahmed Youssef ${ }^{a, b}$, Ricardo L. Carrau ${ }^{b}$, Shahzad Ahmed ${ }^{c}$, Ahmed Tantawy ${ }^{a}$, \\ Ahmed Aly Ibrahima ${ }^{\mathrm{a}}$, Abdulaziz Al Azri ${ }^{\mathrm{C}}$, Mohamed Zahran ${ }^{\mathrm{a}}$
}

\author{
${ }^{a}$ Department of Otolaryngology-Head and Neck \\ Surgery, Alexandria University, Alexandria, \\ Egypt, ' Department of Otolarynglogy-Head \\ and Neck Surgery, OHIO State University \\ Wexner Medical Center, Columbus, Ohio, USA, \\ 'Department of Otolaryngology-Head and Neck \\ Surgery, University Hospital Birmingham, \\ Birmingham, UK \\ Correspondence to Ahmed Sobhy Youssef MD, \\ PhD,MRCS, Head \& Neck/Reconstructive \\ Fellow-University of Newcastle, NSW-Australia \\ 2305; Clinical Fellow Head \& Neck/Skull Base \\ Surgery, University Hospital Birmingham, \\ Birmingham, UK; Lecturer Otolaryngology \& \\ Head and Neck Surgery-Faculty of Medicine- \\ Alexandria University, Egypt, 2131. Tel: +20 \\ 111000 3881; e-mails: \\ ahmedyouseef2002@yahoo.com, Ahmed. \\ Youssef@nhs.net, Ahmed.Youssef@hnehealth. \\ nsw.gov.au
}

Received 23 April 2018

Accepted 25 July 2018

The Egyptian Journal of Otolaryngology 2018, 34:278-282

\begin{abstract}
Background
The endonasal transpterygoid approach has been very popular as a standard approach to the pterygopalatine and infratemporal fossa. However, its implications actually extend beyond these regions to include access to the middle cranial fossa and parapharyngeal space.

Objective

The aim was to illustrate the anatomical landmarks of the endoscopic transpterygoid approach especially the mandibular nerve and clinical implication of this approach through illustrated case presentation.

\section{Materials and methods}

A cadaveric study was performed on three cadaveric adult specimens. Endoscopic medial maxillectomy, Sturman Canefield approach, and completion of transpterygoid approach were done to visualize the different anatomical landmarks, especially the mandibular nerve. An example of clinical application of transpterygoid approach is an illustrated case to biopsy a perineural spread of squamous cell cancer along the mandibular nerve in the lateral wall of cavernous sinus.

\section{Conclusion}

Endoscopic transpterygoid approach is very useful to resect most of benign and some malignant tumors that involve the infratemporal fossa and middle cranial fossa. Good understanding of the V3 anatomy helps in the resection of tumors extending to the infratemporal fossa and poststyloid parapharyngeal space.
\end{abstract}

\section{Keywords:}

infratemporal fossa, internal carotid artery, transpterygoid approach, V2, V3

Egypt J Otolaryngol 34:278-282

(C) 2018 The Egyptian Journal of Otolaryngology

$1012-5574$

\section{Introduction}

Clinical implications of endoscopic endonasal approaches for the management of lesions of the anterior skull base including cribriform, planum sphenoidale, tuberculum sellae, sella, middle and lower clivus, craniovertebral junction, and $\mathrm{C} 1$ have been previously documented. A transpterygoid dissection expands the lateral exposure; thus, gaining access to Meckel's cave, infratemporal fossa, middle cranial fossa, and petrous apex [1-4].

The endoscopic endonasal transpterygoid approach to the infratemporal fossa and middle cranial fossa offers good exposure to the infratemporal fossa, allowing access to larger tumors extending to this area. Moreover, endoscopic endonasal transpterygoid approaches avoid morbidities that are related to other open anterior or lateral approaches such as the cosmetic issues related to skin incision, bony osteotomies or temporalis muscle injury, (temporomandibular joint dysfunction, injuries of facial nerve branches, need for craniotomy, and brain manipulation [5].
The aim of this work was to understand the anatomical landmarks of transpterygoid approach to mandibular nerve and illustrate the importance of this knowledge through case presentation.

\section{Materials and methods}

\section{Cadaveric dissection}

An endoscopic transpterygoid approach was performed in three human anatomic specimens to access the infratemporal fossa and pterygopalatine fossa bilaterally, following institutional protocols. All specimens were injected with red and blue silicone, through the internal carotid artery (ICA) and internal jugular vein, respectively.

Visualization was facilitated by the use of $0^{\circ}, 30^{\circ}$, and $45^{\circ}$ rod lens endoscopes coupled to a high-definition

This is an open access journal, and articles are distributed under the terms of the Creative Commons Attribution-NonCommercial-ShareAlike 4.0 License, which allows others to remix, tweak, and build upon the work non-commercially, as long as appropriate credit is given and the new creations are licensed under the identical terms. 
camera and monitor (Storz Endoscopy, Tuttlingen, Germany). Photos are taken using an HD camera. Surgical dissection was performed using skull base/ neurosurgical endoscopic instruments (Storz), and a high-speed drill with an angled handpiece, and diamond burrs (Total Performance System, Stryker, Kalamazoo, Michigan, USA).

\section{Steps of dissection}

An endoscopic medial maxillectomy was performed by removal of inferior turbinate and all medial wall of the maxillary sinus to the extent of sharply cutting the nasolacrimal duct. Then the approach was completed by removal of the posterior wall of the maxillary antrum from medial to lateral using Kerrison Rongeurs. To reach the most lateral aspect of the infratemporal fossa, the endoscopic Denker's approach was done by making a vertical incision just anterior to the head of the inferior turbinate to reach the edge of the aperture, then dissecting in the subperiosteal plane till reaching the infraorbital foramen that contains its corresponding nerve and vessel.

By dissecting the periosteum and the fat of the pterygopalatine fossa, the main terminal branches of the third part internal maxillary artery were identified including the infraorbital, descending palatine, Vidian, sphenopalatine, and posterior nasal arteries (Fig. 1). Transection of these branches was done to expose the underlying neural structures including the infraorbital, descending palatine, Vidian, and pharyngeal nerves. This portion of the infraorbital nerve accurately delineates the border between infratemporal fossa

Figure 1

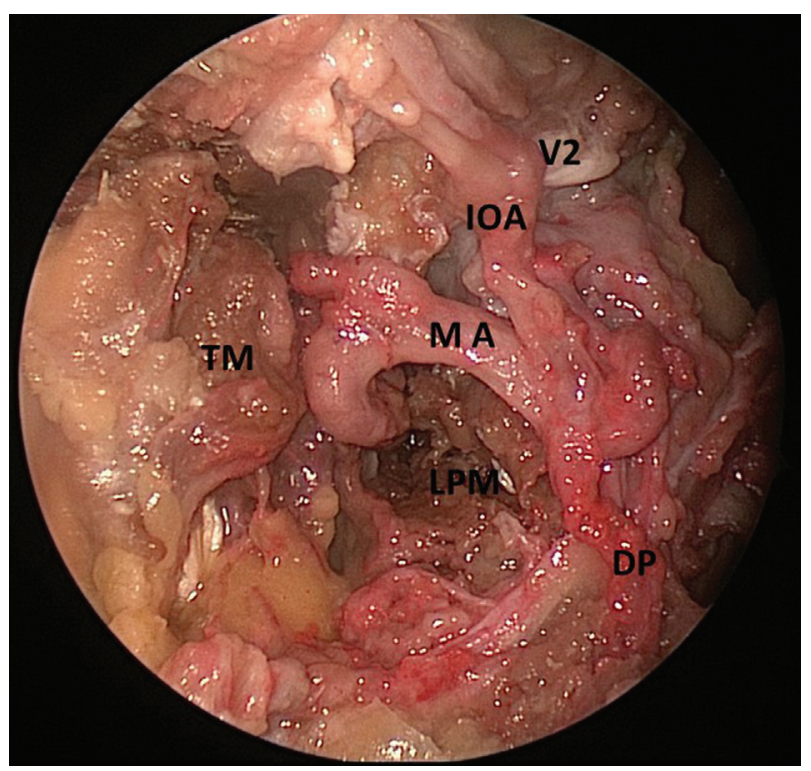

Endoscopic view of right pterygopalatine fossa. and pterygopalatine fossa. We followed the infraorbital nerve medially and posteriorly leading to the foramen rotundum (V2).

Removal of bone from the floor and lateral wall of the sphenoid sinus exposes the bone comprising the floor of the middle cranial fossa, which is the roof of the infratemporal fossa. Dissection of muscles of mastication was done and identification of them by direction of muscle fibers as temporalis muscle fibers is vertically oriented, while the lateral pterygoid muscle is horizontally oriented. The second part of the internal maxillary artery has a variable course, which may run lateral or medial to the lateral pterygoid muscle. The middle meningeal artery (a branch of the first part maxillary artery) may be seen medial to the lateral pterygoid muscle, coursing superiorly toward the foramen spinosum. Resection of the lateral pterygoid muscle and drilling the lateral pterygoid plate improve exposure of the infratemporal fossa, including V3, which lies posterior to the lateral pterygoid plate. Dissection of the mandibular nerve and its divisions was performed and identification of its relationship to other contents in infratemporal fossa like V2 and middle Meningeal artery is illustrated (Figs 2-4).

\section{Case presentation}

A 79-year-old gentleman who has two SCCs removed from his left face. Initially, he had a lesion removed from the left tragal area in 2014. He subsequently had a lesion in the outer aspect of his left eyelid/temple area, which was removed by the dermatologist in November 2016. He describes a gradual droop in the left forehead over the last 6 months.

\section{Figure 2}

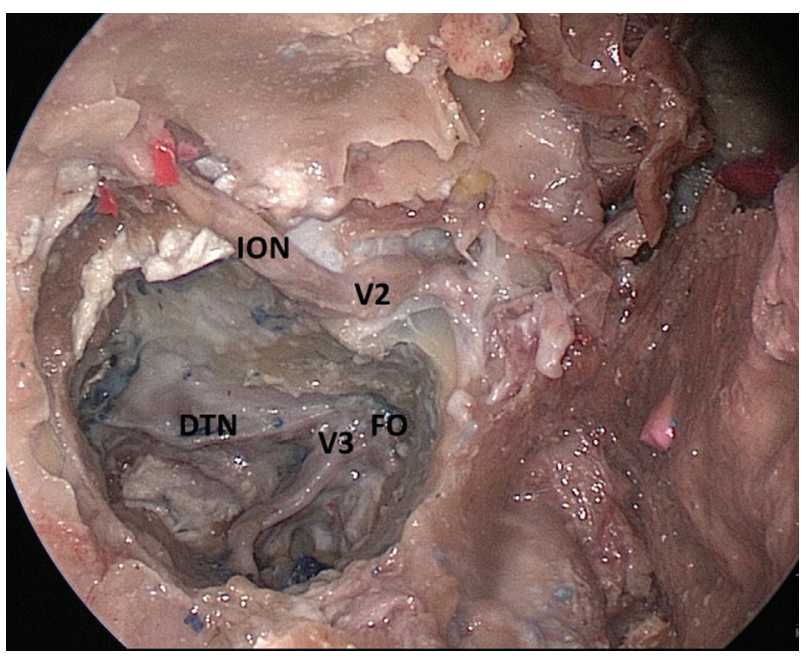

Dissection following the transpterygoid approach to the right infratemporal fossa. 


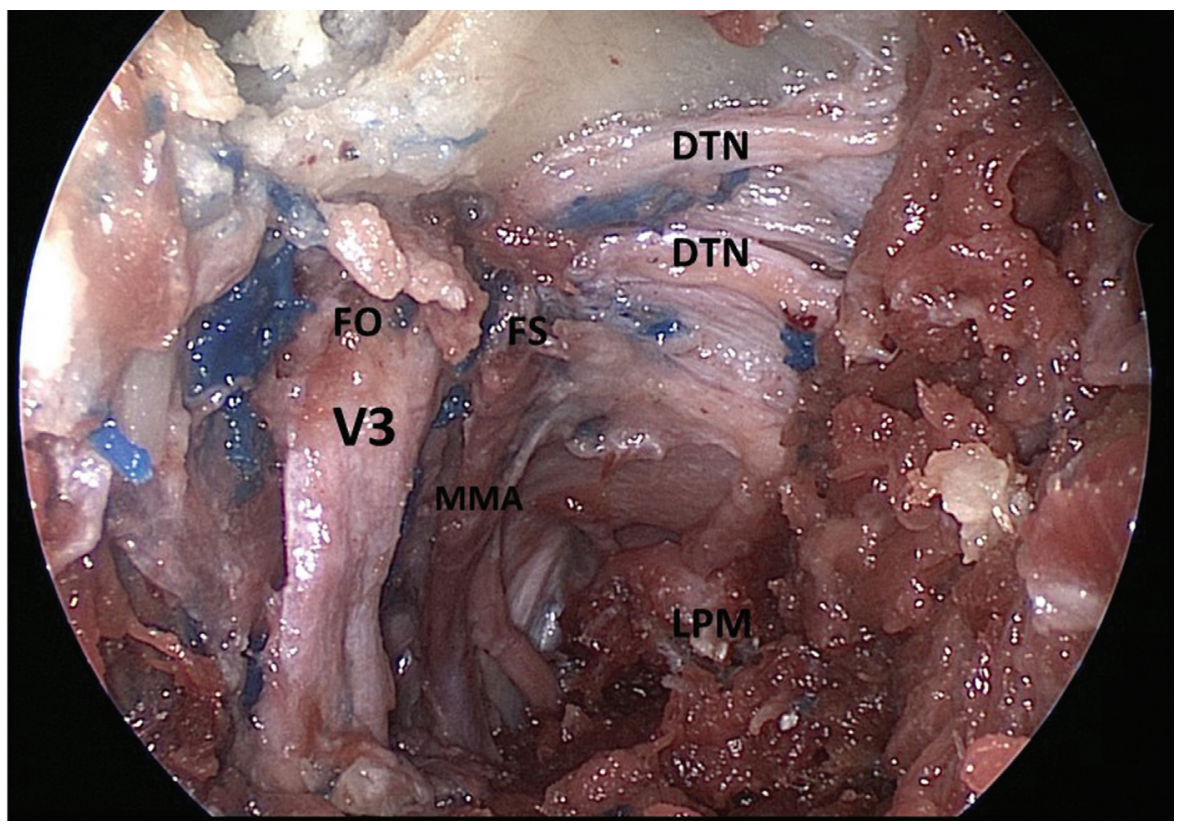

Close view of the left mandibular nerve.

\section{Figure 4}

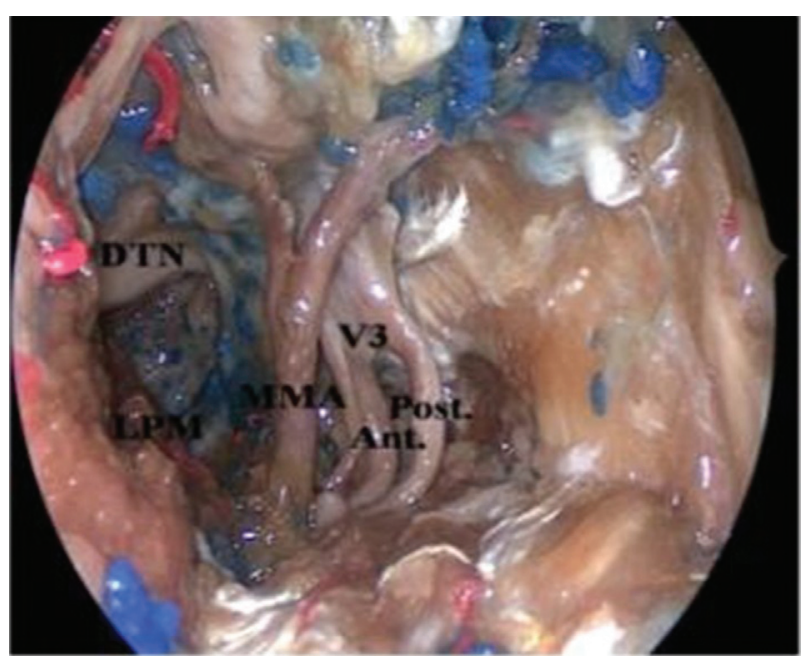

Relations of mandibular nerve in infratemporal fossa.

Since the intervention, he has developed left eye ectropion with watering and numbness of the left lower half of his face and mouth. This has been more pronounced since December 2016.

Initially, this was suspected as Bell's palsy in early January when he reported to the emergency department and he was given a 1 week course of steroids with no improvement. He has also been seen by an oculoplastic surgeon at Birmingham Eye Hospital who has advised him facial reanimation procedures for helping optimal closure of his eyelids.
He was reviewed in the Facial Palsy Clinic in the University Hospital Birmingham with the latest MRI scan done, which has shown a suspicious lesion in the left cavernous sinus area with V3 (mandibular nerve involvement). There is no obvious pathology identified along the facial nerve distribution on the left, but there is evidence of atrophy of the posterior belly of digastric and stylohyoid muscles suggestive of a longstanding facial paresis (Fig. 5).

Clinically, he had a left upper half facial weakness. There was absence of forehead transverse creases. He applied maximal effort to close his left eye. There was obvious epiphora and ectropion of the lower eyelid with bogginess around the lower eyelid. There was no evidence of any clinical recurrence of skin cancer at the two previous excision sites.

He had obvious numbness along the mandibular nerve distribution and also there was a difficulty in mastication and taste on the left. His ocular movements were good and his visual acuity was appropriate suggesting that the $2,3,4$, and 6 cranial nerves were functioning optimally. He also had good function in his $9,10,11$, and 12 cranial nerves. He never experienced any symptoms suggesting any obvious otological pathology or vestibular symptoms on examination.

We had reviewed his scans in the Skull Base MDT of University Hospital Birmingham to make a discussion with regard to the suspicious lesion, which had been picked up in the latest scan in his left cavernous sinus. 
The decision was taken to biopsy that lesion. Consequently, a transpterygoid approach with the same steps like cadaveric dissection was done and biopsy confirmed malignant squamous cells, suggesting a possible perineural spread of original squamous cell carcinoma of the face and the patient was referred for intensity-modulated radiotherapy of involved V3 and lateral wall of left cavernous sinus.

\section{Discussion}

Various lateral and anterior approaches have been described to access the infratemporal and middle cranial fossae. Lateral approaches include a combination of preauricular or postauricular incisions, parotidectomy with facial nerve rerouting and preservation, resection of the mandibular condyle, bony osteotomies, displacement of the temporalis muscle with or without coronoid resection, dissection transposition of the petrous ICA, and pterional craniotomy [6].

In comparison to open approaches to the coronal plane, the endoscopic transpterygoid approach provides better exposure of the nasopharynx, eustachian tube, and central skull base. In addition, it provides excellent exposure of other key landmarks like foramen rotundum, V2, vidian canal, foramen ovale, and V3 by drilling of lateral pterygoid plate and dissection of lateral pterygoid muscle. Some dental or palatine branches of V2 are often sacrificed resulting in numbness and sensory loss to the ipsilateral palate and the upper jaw $[6,7]$.

The mandibular nerve, after coming out from the skull base through the foramen ovale lies on the lateral surface of the eustachian tube that runs from the middle ear and descends inferiorly, anteriorly, and medially to open into the nasopharynx. The intimate relationship between the ICA, the eustachian tube, and $\mathrm{V} 3$ is well described in addition to the role of the eustachian tube as a key landmark to the superior portion of the parapharyngeal ICA and the jugular foramen [8-10].

The endoscopic endonasal transpterygoid approach has been utilized in other indications in endoscopic skull base surgery like the transposition of a temporoparietal fascia to reconstruct a skull base defect or to access the lateral recess of the sphenoid sinus like cerebrospinal fluid leaks resulting from persistent Sternberg's canal or to reach the petrous apex and Meckel's cave or during endoscopic nasopharyngectomy $[7,10]$.The presented case was a suspected lesion along V3 that was centered along the nerve exit from the foramen ovale that was approached by endoscopic transpterygoid approach by drilling of the pterygoid wedge and exposure of the Vidian nerve, ICA, and middle fossa dura to make a formal confirmatory diagnosis of this lesion that proved later to be a perineural spread of squamous cell carcinoma. Then the patient underwent chemoradiotherapy without any delay which showed that the endoscopic approach was the most feasible one. Alternative options include open approaches that lack good visualization and leave patients with severe morbidities like possible craniotomy or (temporomandibular joint dysfunction.

\section{Conclusion}

Endonasal endoscopic transpterygoid approaches have been very popular for the treatment of lesions affecting the infratemporal fossa. It could be also utilized to approach lesions in difficult regions like the parapharyngeal space, lateral wall of cavernous sinus, and middle cranial fossa. Understanding these landmarks avoid unnecessary open approaches that lead to significant comorbidities and facial scars.

\section{Acknowledgements}

The article was accepted as a poster presentation in AAO-HNS September 2017 and was presented in British Skull Base Society in January 2017.

\section{Financial support and sponsorship \\ Nil.}

\section{Conflicts of interest}

There are no conflicts of interest.

\section{References}

1 Kassam AB, Gardner P, Snyderman C, Mintz A, Carrau R. Expanded endonasal approach: fully endoscopic, completely transnasal approach to the middle third of the clivus, petrous bone, middle cranial fossa, and infratemporal fossa. Neurosurg Focus 2005; 19:E6.

2 Fortes FS, Sennes LU, Carrau RL, et al. Endoscopic anatomy of the pterygopalatine fossa and the transpterygoid approach: development of a surgical instruction model. Laryngoscope 2008; 118:44-49.

3 Kassam AB, Prevedello DM, Carrau RL, et al. The front door to Meckel's cave: an anteromedial corridor via expanded endoscopic endonasal approach - technical considerations and clinical series. Neurosurgery 2009; 64:71-82.

4 Zanation AM, Snyderman CH, Carrau RL, et al. Endoscopic endonasal surgery for petrous apex lesions. Laryngoscope 2009; 119:19-25.

5 Mousa Sadr Hosseini S, Razfar A, Carrau RL, et al. Endonasal transpterygoid approach to the infratemporal fossa: correlation of endoscopic and multiplanar CT anatomy. Head Neck 2012; 34:313-320.

6 Vilela MD, Rostomily RC. Temporomandibular joint-preserving preauricular subtemporal-infratemporal fossa approach: surgical technique and clinical application. Neurosurgery 2004; 55:143-153; discussion 153-154.

7 Kasemsiri P, Solares CA, Carrau RL, et al. Endoscopic endonasal transpterygoid approaches: anatomical landmarks for planning the surgical corridor. Laryngoscope 2013; 123:811-815. 
8 Vescan AD, Snyderman CH, Carrau RL, et al. Vidian canal: analysis and relationship to the internal carotid artery. Laryngoscope 2007; 117:1338-1342.

9 Kassam AB, Vescan AD, Carrau RL, et al. Expanded endonasal approach: Vidian canal as a landmark to the petrous internal carotid artery. J Neurosurg 2008; 108:177-183.
10 Dallan I, Lenzi R, Bignami M, Battaglia P, Sellari-Franceschini S, Muscatello L, et al. Endoscopic transnasal anatomy of the infratemporal fossa and upper parapharyngeal regions: correlations with traditional perspectives and surgical implications. Minim Invasive Neurosurg 2010; 53:261-269. 